\title{
THE LATEST TRENDS IN CLINICAL TRANSPLANTATION ON THE RESULTS OF THE WORLD CONGRESS OF TRANSPLANTATION (26-31.07.2014, SAN FRANCISCO, USA)
}

The World Congress of Transplantation was held on 26.0731.07.2014 in San Francisco (USA). There were presented 3090 reports, among them $2073(70 \%)$ were in clinical transplantation, including renal transplantation - 1,089 reports, liver transplantation - 495, heart transplantation - 71 , lungs transplantation -35 , and pancreas transplantation - 11. An overall increase in transplant activity was observed, especially significant in a number of African countries (Tunisia, Sudan, and Nigeria), Thailand, Qatar, Iran, Brazil and a significant improvement in the results of transplantation, promoting longevity of transplant recipients with good quality of life. As one of many examples can be childbirth in women with a heart transplanted in early childhood.

Almost everywhere, a significant intensification of transplantation activities in a short time was provided at the government level as part of the standard and absolutely affordable technologies: adaptation of specialists and the public to perceive the concept of brain death as the criterion of a person's death, organization of transplant coordination service, motivation increase in the implementation of the donor process. There is a significant increase in related donation by a pair organs exchange (first informed of 1000 kidney transplants in the United States) and transplantation of organs from donors with blood group incompatibility (more than 1,000 transplants of kidneys in Japan).

Development of clinical transplantation has led to a change in standards towards a significant expansion of the indications for kidney transplantation and donation. There are not any age limits for renal, liver, heart and lungs transplantation in both a donor and a recipient. Opportunities of transplantation in patients with HIV and hepatitis viral infections, as well as multiple organ failure, have increased. There appeared a group of new highly effective drugs for the treatment of viral hepatitis C (Sofosbuvir, Simeprevir et al.), that eliminate the need for interferon in transplant candidates and recipients.

There were expanded indications for the use of the «problem organs» taken from donors with asystolia and various kidney diseases (tumors, urolithiasis, polycystic) or from social risk groups, and even after euthanasia.

Minimally invasive technologies are being introduced. Laparoscopic donor nephrectomy has become the standard because of the proven advantages compared to open surgery. Laparoscopic donor resections are being performed on the pancreas and the liver. There were performed dozens of laparoscopic kidney transplants using robotics. The most minimally invasive laparoscopic kidney transplantation is recognized the transplantation through the vagina. At the Congress there was represented the first successful experience of transplantation of the uterus. There was continued an extensive use of machine-cold perfusion of the kidneys, especially in the preservation of kidneys from older donors and asystole. There started the use of normothermic perfusion, which has allowed providing the primary function of kidney transplants, unapproved in the framework of existing standards for transplantation.

With regard to immunosuppression, there was a trend to increase the use of antibodies anti-CD25 (Simulect), anti-CD20 (Rituximab), antiCD52 (Campath-1H) at the stage of induction and testing of a wide range of antibodies to different parts of the immune system (Eculizumab - antiC5a, Tocilizumab - anti-II-6R, Berinert - esterase inhibitor $C$ and others) involved in the development of rejection, in order to achieve minimal but adequate immunosuppression in the absence of complications, and in the long-term - induction of transplantation tolerance. That led to a sharp reduction in the number of rejection crises and refusal of the steroids use in $40 \%$ of patients. Information about the possibility of eliminating the use of calcineurin inhibitors, including Advagraf, were not given, but effective protocols of their use minimization were presented.

The materials of the Congress also clearly demonstrated that the most significant for the further development of transplantation is a broad discussion in society of the ethical, legal and psychosocial aspects of transplantation, training for professionals and improve of the donor process organization.

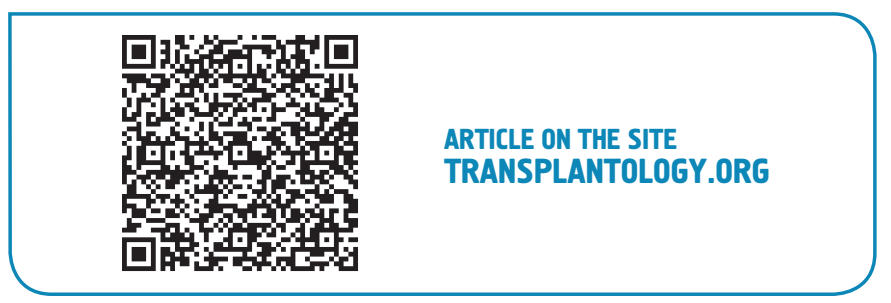

The author indicates no potential conflicts of interest. 